\title{
Fast convergences towards Euler-Mascheroni constant
}

\section{CRISTINEL MORTICI}

Valahia University of Târgovişte, Department of Mathematics, Bd. Unirii 18, 130082

Târgovişte, Romania

E-mails: cmortici@valahia.ro / cristinelmortici@yahoo.com

\begin{abstract}
The aim of this paper is to introduce a new family of sequences which faster converge
\end{abstract} to the Euler-Mascheroni constant. Finally, numerical computations are given.

Mathematical subject classification: 41A60, 41A25, 57Q55.

Key words: Euler-Mascheroni constant; speed of convergence.

\section{Introduction}

One of the most important constants in mathematics is defined as the limit of the sequence

$$
\gamma_{n}=1+\frac{1}{2}+\frac{1}{3}+\cdots+\frac{1}{n}-\ln n,
$$

denoted $\gamma=0.57721566490153286 \ldots$. It is now known as the Euler-Mascheroni constant, in honour of the Swiss mathematician Leonhard Euler (17071783) and of the Italian mathematician Lorenzo Mascheroni (1750-1800).

The sequence $\left(\gamma_{n}\right)_{n \geq 1}$ and the constant $\gamma$ have numerous applications in many areas of mathematics, such as analysis, theory of probability, special functions, and number theory. As a consequence, many authors are preoccupied to improve the speed of convergence of the sequence $\left(\gamma_{n}\right)_{n \geq 1}$, which is very slowly, if we take into account that it converges toward its limit like $n^{-1}$. 
More precisely, we mention the following results related to the speed of convergence of the sequence $\left(\gamma_{n}\right)_{n \geq 1}$ :

$$
\frac{1}{2(n+1)}<\gamma_{n}-\gamma<\frac{1}{2 n} \quad \text { (Young) }
$$

(see, [14, 15, 28]). We also refer here to the papers [1, 2, 5-12, 20-27], where important improvements of the speed of convergence of $\gamma_{n}$ were established.

The complete asymptotic expansion of the sequence $\left(\gamma_{n}\right)_{n \geq 1}$ is

$$
\gamma_{n} \sim \gamma+\frac{1}{2 n}-\sum_{k=1}^{\infty} \frac{B_{2 k}}{2 k} \frac{1}{n^{2 k}}
$$

where the Bernoulli numbers $B_{2 k}$ are defined by

$$
\frac{t}{e^{t}-1}=\sum_{k=0}^{\infty} \frac{B_{k}}{k !} t^{k}
$$

As $B_{2}=1 / 6, B_{4}=-1 / 30, B_{6}=1 / 42, B_{8}=-1 / 30$, we imply

$$
\gamma_{n} \sim \gamma+\frac{1}{2 n}-\frac{1}{12 n^{2}}+\frac{1}{120 n^{4}}-\frac{1}{252 n^{6}}+\frac{1}{240 n^{8}}-\cdots .
$$

DeTemple [3-4] introduced the sequence

$$
R_{n}=1+\frac{1}{2}+\frac{1}{3}+\cdots+\frac{1}{n}-\ln \left(n+\frac{1}{2}\right)
$$

which converges to $\gamma$ like $n^{-2}$, since

$$
\frac{1}{24(n+1)^{2}}<R_{n}-\gamma<\frac{1}{24 n^{2}} .
$$

Recently, Mortici [17] introduced the sequences

$$
u_{n}=1+\frac{1}{2}+\frac{1}{3}+\cdots+\frac{1}{n-1}+\frac{1}{(6-2 \sqrt{6}) n}-\ln \left(n+\frac{1}{\sqrt{6}}\right)
$$

and

$$
v_{n}=1+\frac{1}{2}+\frac{1}{3}+\cdots+\frac{1}{n-1}+\frac{1}{(6+2 \sqrt{6}) n}-\ln \left(n-\frac{1}{\sqrt{6}}\right),
$$


which converges as $n^{-3}$, since

$$
\lim _{n \rightarrow \infty} n^{3}\left(u_{n}-\gamma\right)=-\frac{\sqrt{6}}{108} \quad \text { and } \quad \lim _{n \rightarrow \infty} n^{3}\left(v_{n}-\gamma\right)=\frac{\sqrt{6}}{108} .
$$

See [17, Theorem 2.1]. Furthermore, the arithmetic mean of the sequences $\left(u_{n}\right)_{n \geq 1}$ and $\left(v_{n}\right)_{n \geq 1}$,

$$
z_{n}=1+\frac{1}{2}+\frac{1}{3}+\cdots+\frac{1}{n-1}+\frac{1}{2 n}-\frac{1}{2} \ln \left(n^{2}-\frac{1}{6}\right)
$$

converges to $\gamma$ as $n^{-4}$.

We open here a new direction to accelerate the sequence $\left(\gamma_{n}\right)_{n \geq 1}$, that is to consider an additional term of the form

$$
M_{n}=\gamma_{n}-\gamma+\ln \frac{P(n)}{Q(n)},
$$

where $P, Q$ are polynomials of the same degree, having the leading coefficient equal to one. Precisely, we introduce the sequences

$$
v_{n}=\gamma_{n}+\ln \frac{n-\frac{1}{12}}{n+\frac{5}{12}} \quad \text { and } \quad \mu_{n}=\gamma_{n}+\ln \frac{n^{2}+\frac{33}{140} n+\frac{37}{1680}}{n^{2}+\frac{103}{140} n+\frac{61}{336}}
$$

whose speeds of convergence increase to $n^{-3}$, respective $n^{-5}$, since

$$
\lim _{n \rightarrow \infty} n^{3}\left(v_{n}-\gamma\right)=\frac{-7}{288} \quad \text { and } \quad \lim _{n \rightarrow \infty} n^{5}\left(\mu_{n}-\gamma\right)=\frac{3959}{806400} .
$$

Our study is based on the following result, which represents a powerful tool for constructing some asymptotic expansions, or to accelerate some convergences.

Lemma 1. If $\left(\omega_{n}\right)_{n \geq 1}$ is convergent to zero and there exists the limit

$$
\lim _{n \rightarrow \infty} n^{k}\left(\omega_{n}-\omega_{n+1}\right)=l \in[-\infty, \infty],
$$

with $k>1$, then there exists the limit:

$$
\lim _{n \rightarrow \infty} n^{k-1} \omega_{n}=\frac{l}{k-1} .
$$

For proofs and further applications, see [13-19]. The sequences (1)-(2) were introduced in [17] using Lemma 1. Clearly the sequence $\left(\omega_{n}\right)_{n \geq 1}$ converges more quickly when the value of $k$ satisfying (3) is larger. 


\section{First degree term}

In this section we define the sequence

$$
\omega_{n}=\gamma_{n}-\gamma+\ln \frac{n+a}{n+b}
$$

to find the values $a, b$ which provide the fastest sequence $\left(\omega_{n}\right)_{n \geq 1}$. First

$$
\omega_{n}-\omega_{n+1}=-\frac{1}{n+1}-\ln \frac{n}{n+1}+\ln \frac{n+a}{n+b}-\ln \frac{n+1+a}{n+1+b},
$$

and we are concentrated to compute a limit of the form (3). In this sense, we used a computer software to obtain the following representation in power series:

$$
\begin{gathered}
\omega_{n}-\omega_{n+1}=\left(a-b+\frac{1}{2}\right) \frac{1}{n^{2}}+\left(-a^{2}-a+b^{2}+b-\frac{2}{3}\right) \frac{1}{n^{3}} \\
+\left(a^{3}+\frac{3}{2} a^{2}+a-b^{3}-\frac{3}{2} b^{2}-b+\frac{3}{4}\right) \frac{1}{n^{4}}+O\left(\frac{1}{n^{5}}\right) .
\end{gathered}
$$

We can state the following

\section{Theorem 2.}

i) If $a-b+\frac{1}{2} \neq 0$, then the speed of convergence of the sequence $\left(\omega_{n}\right)_{n \geq 1}$ is $n^{-1}$, since

$$
\lim _{n \rightarrow \infty} n^{2}\left(\omega_{n}-\omega_{n+1}\right)=a-b+\frac{1}{2} \text { and } \lim _{n \rightarrow \infty} n \omega_{n}=a-b+\frac{1}{2} \neq 0 .
$$

ii) If $a-b+\frac{1}{2}=0$ and $-a^{2}-a+b^{2}+b-\frac{2}{3} \neq 0$, then the speed of convergence of the sequence $\left(\omega_{n}\right)_{n \geq 1}$ is $n^{-2}$, since

$$
\lim _{n \rightarrow \infty} n^{3}\left(\omega_{n}-\omega_{n+1}\right)=-a^{2}-a+b^{2}+b-\frac{2}{3}
$$

and

$$
\lim _{n \rightarrow \infty} n^{2} \omega_{n}=\frac{1}{2}\left(-a^{2}-a+b^{2}+b-\frac{2}{3}\right) \neq 0 .
$$

iii) If $a-b+\frac{1}{2}=0$ and $-a^{2}-a+b^{2}+b-\frac{2}{3}=0$ (equivalent with $a=$ $-1 / 12, b=5 / 12)$, then the speed of convergence of the sequence $\left(\omega_{n}\right)_{n \geq 1}$ is $n^{-3}$, since

$$
\lim _{n \rightarrow \infty} n^{4}\left(\omega_{n}-\omega_{n+1}\right)=\frac{-7}{96} \text { and } \lim _{n \rightarrow \infty} n^{3} \omega_{n}=\frac{-7}{288} .
$$


The proof of Theorem 2 easily follows from Lemma 1 and (4).

For $a=-1 / 12, b=5 / 12$, the relation (4) becomes

$$
\omega_{n}-\omega_{n+1}=-\frac{7}{96 n^{4}}+O\left(\frac{1}{n^{5}}\right)
$$

and so, iii) is completely proved.

\section{Second degree term}

Now we define the sequence

$$
\lambda_{n}=\gamma_{n}-\gamma+\ln \frac{n^{2}+a n+b}{n^{2}+c n+d}
$$

to find the values $a, b, c, d$ which provide the fastest sequence $\left(\lambda_{n}\right)_{n \geq 1}$. First

$$
\begin{aligned}
\lambda_{n}-\lambda_{n+1}= & -\frac{1}{n+1}-\ln \frac{n}{n+1}+\ln \frac{n^{2}+a n+b}{n^{2}+c n+d} \\
& -\ln \frac{(n+1)^{2}+a(n+1)+b}{(n+1)^{2}+c(n+1)+d},
\end{aligned}
$$

and we are concentrated to compute a limit of the form (3).

In this sense, we used again the computer software to obtain the following representation in power series:

$$
\begin{gathered}
\lambda_{n}-\lambda_{n+1}=\left(a-c+\frac{1}{2}\right) \frac{1}{n^{2}}-\left(a^{2}+a-c^{2}-c-2 b+2 d+\frac{2}{3}\right) \frac{1}{n^{3}} \\
+\left(a-3 b-c+3 d-3 a b+3 c d+\frac{3}{2} a^{2}+a^{3}-\frac{3}{2} c^{2}-c^{3}+\frac{3}{4}\right) \frac{1}{n^{4}} \\
-\left(a^{4}+2 a^{3}-4 a^{2} b+2 a^{2}-6 a b+a+2 b^{2}-4 b-c^{4}\right. \\
\left.-2 c^{3}+4 c^{2} d-2 c^{2}+6 c d-c-2 d^{2}+4 d+\frac{4}{5}\right) \frac{1}{n^{5}} \\
+\left(a-5 b-5 a^{3} b+5 d-c-\frac{5}{2} c^{2}-\frac{10}{3} c^{3}-\frac{5}{2} c^{4}-c^{5}+\frac{5}{2} a^{4}\right. \\
+10 c d-5 d^{2}+10 c^{2} d+5 c^{3} d-5 c d^{2}+a^{5}+5 a b^{2} \\
\left.-10 a b+5 b^{2}+\frac{10}{3} a^{3}-10 a^{2} b+\frac{5}{2} a^{2}+\frac{5}{6}\right) \frac{1}{n^{6}}+O\left(\frac{1}{n^{7}}\right) .
\end{gathered}
$$

We can state the following 


\section{Theorem 3.}

i) Let us denote the coefficients of (5) by

$$
\begin{aligned}
\alpha= & a-c+\frac{1}{2} \\
\beta= & -\left(a^{2}+a-c^{2}-c-2 b+2 d+\frac{2}{3}\right) \\
\delta= & a-3 b-c+3 d-3 a b+3 c d+\frac{3}{2} a^{2}+a^{3}-\frac{3}{2} c^{2}-c^{3}+\frac{3}{4} \\
\eta= & -\left(a^{4}+2 a^{3}-4 a^{2} b+2 a^{2}-6 a b+a+2 b^{2}-4 b-c^{4}\right. \\
& \left.-2 c^{3}+4 c^{2} d-2 c^{2}+6 c d-c-2 d^{2}+4 d+\frac{4}{5}\right)
\end{aligned}
$$

ii) If $\alpha \neq 0$, then the speed of convergence of the sequence $\left(\lambda_{n}\right)_{n \geq 1}$ is $n^{-1}$, since

$$
\lim _{n \rightarrow \infty} n^{2}\left(\lambda_{n}-\lambda_{n+1}\right)=\alpha \text { and } \lim _{n \rightarrow \infty} n \lambda_{n}=\alpha \neq 0 .
$$

iii) If $\alpha=0$ and $\beta \neq 0$, then the speed of convergence of the sequence $\left(\lambda_{n}\right)_{n \geq 1}$ is $n^{-2}$, since

$$
\lim _{n \rightarrow \infty} n^{3}\left(\lambda_{n}-\lambda_{n+1}\right)=\beta \quad \text { and } \quad \lim _{n \rightarrow \infty} n^{2} \lambda_{n}=\frac{\beta}{2} \neq 0 .
$$

iv) If $\alpha=\beta=0$ and $\delta \neq 0$, then the speed of convergence of the sequence $\left(\lambda_{n}\right)_{n \geq 1}$ is $n^{-3}$, since

$$
\lim _{n \rightarrow \infty} n^{4}\left(\lambda_{n}-\lambda_{n+1}\right)=\delta \text { and } \lim _{n \rightarrow \infty} n^{3} \lambda_{n}=\frac{\delta}{3} \neq 0 .
$$

v) If $\alpha=\beta=\delta=0$ and $\eta \neq 0$, then the speed of convergence of the sequence $\left(\lambda_{n}\right)_{n \geq 1}$ is $n^{-4}$, since

$$
\lim _{n \rightarrow \infty} n^{5}\left(\lambda_{n}-\lambda_{n+1}\right)=\eta \text { and } \quad \lim _{n \rightarrow \infty} n^{4} \lambda_{n}=\frac{\eta}{4} \neq 0 .
$$

vi) If $a=\beta=\delta=\eta=0$ (equivalent with $a=33 / 140, b=37 / 1680$, $c=103 / 140, d=61 / 336)$, then the speed of convergence of the sequence $\left(\lambda_{n}\right)_{n \geq 1}$ is $n^{-5}$, since

$$
\lim _{n \rightarrow \infty} n^{6}\left(\lambda_{n}-\lambda_{n+1}\right)=\frac{3959}{161280} \text { and } \lim _{n \rightarrow \infty} n^{5} \lambda_{n}=\frac{3959}{806400} .
$$


The proof of Theorem 3 easily follows from Lemma 1 and (5).

For $a=33 / 140, b=37 / 1680, c=103 / 140, d=61 / 336$, the relation (5) becomes

$$
\lambda_{n}-\lambda_{n+1}=\frac{3959}{161280 n^{6}}+O\left(\frac{1}{n^{7}}\right) .
$$

and so, vi) is completely proved.

\section{Concluding remarks}

As least theoretically, further sequences of the form

$$
M_{n}=\gamma_{n}-\gamma+\ln \frac{P(n)}{Q(n)}
$$

can be defined, where $\operatorname{deg} P=\operatorname{deg} Q=k \geq 3$. As above,

$$
M_{n}-M_{n+1}=-\frac{1}{n+1}-\ln \frac{n}{n+1}+\ln \frac{P(n) Q(n+1)}{Q(n) P(n+1)}
$$

and if we expand (6) into a power series of $n^{-1}$, then the $2 k$ coefficients of the polynomials $P$ and $Q$ are the unique solution of the system obtained by imposing that the first $2 k$ coefficients of the power series (6) vanish. In this case,

$$
M_{n}-M_{n+1}=\frac{\theta}{n^{2 k+2}}+O\left(\frac{1}{n^{2 k+3}}\right),
$$

with $\theta \neq 0$. By Lemma $1,\left(M_{n}\right)_{n \geq 1}$ tends to zero as $n^{-(2 k+1)}$, since

$$
\lim _{n \rightarrow \infty} n^{2 k+1} M_{n}=\frac{\theta}{2 k+1} .
$$

Finally, we offer some numerical computations to prove the superiority of our sequences $\left(v_{n}\right)_{n \geq 1}$ and $\left(\mu_{n}\right)_{n \geq 1}$ over the classical sequence $\left(\gamma_{n}\right)_{n \geq 1}$ and the DeTemple sequence $\left(R_{n}\right)_{n \geq 1}$. Remark that already $\mu_{1}$ approximates $\gamma$ with seven exact decimals.

\begin{tabular}{|c|c|c|c|c|}
\hline$n$ & $\gamma_{n}-\gamma$ & $R_{n}-\gamma$ & $\gamma-v_{n}$ & $\mu_{n}-\gamma$ \\
\hline 10 & $4.9167 \times 10^{-2}$ & $3.7733 \times 10^{-4}$ & $2.2748 \times 10^{-5}$ & $\mathbf{4 . 3 2 3 7 \times \mathbf { 1 0 } ^ { - 8 }}$ \\
\hline 50 & $9.9667 \times 10^{-3}$ & $1.6337 \times 10^{-5}$ & $1.9192 \times 10^{-7}$ & $\mathbf{1 . 5 3 3 0 \times \mathbf { 1 0 } ^ { - 1 1 }}$ \\
\hline 100 & $4.9917 \times 10^{-3}$ & $4.1252 \times 10^{-6}$ & $2.4147 \times 10^{-8}$ & $\mathbf{4 . 8 4 9 9} \times \mathbf{1 0}^{-13}$ \\
\hline 300 & $1.6657 \times 10^{-3}$ & $4.6142 \times 10^{-7}$ & $8.9825 \times 10^{-10}$ & $\mathbf{2 . 0 1 2 2} \times \mathbf{1 0}^{-15}$ \\
\hline
\end{tabular}


Our approach works by small degrees $k$ of $P(t)$ and $Q(t)$, respectively, compared with the obtained order $2 k+1$ of the speed of convergence, but for larger values of $k$, it becomes quite difficult (even for computer softwares) to compute the coefficients of the polynomials and the limit $\theta /(2 k+1)$ of the sequences $n^{2 k+1} M_{n}$.

We propose now a refined numerical method to compute the coefficients based on an explicit formula for the series expansion of $M_{n}-M_{n+1}$ around $1 / n$.

Starting with formula (6), we have

$$
M_{n}-M_{n+1}=-\frac{1}{n+1}-\ln \frac{n}{n+1}+\ln \frac{P(n)}{Q(n)}-\ln \frac{P(n+1)}{Q(n+1)} .
$$

As in the cases studied above, we assume that the polynomials $P$ and $Q$ have rational coefficients. Let us consider the factorizations

$$
\begin{gathered}
P(t)=t^{k}+a_{k-1} t^{k-1}+\cdots+a_{0}=\left(t-\alpha_{1}\right)\left(t-\alpha_{2}\right) \cdots\left(t-\alpha_{k}\right) \\
Q(t)=t^{k}+b_{k-1} t^{k-1}+\cdots+b_{0}=\left(t-\beta_{1}\right)\left(t-\beta_{2}\right) \cdots\left(t-\beta_{k}\right),
\end{gathered}
$$

where the roots $\alpha_{v}$ and $\beta_{v}$ are complex numbers. Then (7) takes the form

$$
M_{n}-M_{n+1}=-\frac{1}{n+1}-\ln \frac{n}{n+1}+\sum_{j=1}^{k}\left(\ln \frac{n-\alpha_{j}}{n-\beta_{j}}-\ln \frac{n-\left(\alpha_{j}-1\right)}{n-\left(\beta_{j}-1\right)}\right) .
$$

Next, we have for real numbers $\alpha, \beta$ that

$$
\begin{aligned}
\ln \frac{n-\alpha}{n-\beta} & =\ln \left(1+\frac{\beta-\alpha}{n-\beta}\right)=\sum_{\nu=1}^{\infty} \frac{(-1)^{\nu-1}}{v}\left(\frac{\beta-\alpha}{n-\beta}\right)^{\nu} \\
& =\sum_{\nu=1}^{\infty} \frac{(-1)^{\nu-1}}{v}\left(\frac{\beta-\alpha}{n}\right)^{\nu} \frac{1}{(1-\beta / n)^{v}} \\
& =\sum_{\nu=1}^{\infty} \frac{(-1)^{\nu-1}(\beta-\alpha)^{\nu}}{v n^{\nu}} \sum_{\mu=0}^{\infty}\left(\begin{array}{c}
v+\mu-1 \\
v-1
\end{array}\right)\left(\frac{\beta}{n}\right)^{\mu} \\
& =-\sum_{d=1}^{\infty}\left(\sum_{\nu=1}^{d}\left(\begin{array}{c}
d-1 \\
v-1
\end{array}\right) \frac{(\alpha-\beta)^{v} \beta^{d-v}}{v}\right) \frac{1}{n^{d}} \quad(\text { with } d=v+\mu) \\
& =-\sum_{d=1}^{\infty} \frac{\alpha^{d}-\beta^{d}}{d n^{d}} .
\end{aligned}
$$


Replacing $\alpha$ by $\alpha-1$ and $\beta$ by $\beta-1$, we get

$$
\ln \frac{n-(\alpha-1)}{n-(\beta-1)}=-\sum_{d=1}^{\infty} \frac{(\alpha-1)^{d}-(\beta-1)^{d}}{d n^{d}}
$$

and

$$
\ln \frac{n}{n+1}=\sum_{d=1}^{\infty} \frac{(-1)^{d}}{d n^{d}}
$$

Finally, using

$$
\frac{1}{n+1}=\sum_{d=1}^{\infty} \frac{(-1)^{d-1}}{n^{d}}
$$

it follows from (10)-(13):

$$
\begin{gathered}
M_{n}-M_{n+1}=\sum_{d=2}^{\infty} \\
\times\left((-1)^{d}(d-1)+\sum_{j=1}^{k}\left(\left(\alpha_{j}-1\right)^{d}-\alpha_{j}^{d}\right)\left(\left(\beta_{j}-1\right)^{d}-\beta_{j}^{d}\right)\right) \frac{1}{d n^{d}} .
\end{gathered}
$$

For every $d$ the corresponding term in brackets on the right-hand side of (14) is a polynomial in $2 k$ variables, which is symmetric in $\alpha_{1}, \alpha_{2}, \ldots, \alpha_{k}$ and in $\beta_{1}, \beta_{2}, \ldots, \beta_{k}$. Therefore, the proof of the main theorem on symmetric polynomials involves an algorithm to express the polynomials (14) in terms of the elementary symmetric polynomials in $\alpha_{1}, \alpha_{2}, \ldots, \alpha_{k}$ and in $\beta_{1}, \beta_{2}, \ldots, \beta_{k}$. So, one obtains equations in terms of the coefficients $a_{0}, \ldots, a_{k}$ and $b_{0}, \ldots, b_{k}$.

But, a second method to deduce the rational coefficients $a_{0}, \ldots, a_{k}$ and $b_{0}, \ldots, b_{k}$ from the (complex) solution of the system

$$
\begin{gathered}
(-1)^{d}(d-1)+\sum_{j=1}^{k} \\
\times\left(\left(\alpha_{j}-1\right)^{d}-\alpha_{j}^{d}\right)\left(\left(\beta_{j}-1\right)^{d}-\beta_{j}^{d}\right)=0 \quad(2 \leq d \leq 2 k+1)
\end{gathered}
$$

is based on the numerical continued fraction algorithm. Provided that the coefficients $a_{0}, \ldots, a_{k-1}$ and $b_{0}, \ldots, b_{k-1}$ are rationals, the following pure numerical method works without using any computer algebra software in order to obtain the coefficients. Here, we first solve the system (15) numerically, 
which for large $k$ is much simpler than to compute the coefficients $a_{0}, \ldots, a_{k}$ and $b_{0}, \ldots, b_{k}$ by a computer algebra system. We demonstrate the method by computing the rationals $a_{0}, a_{1}, a_{2}$ and $b_{0}, b_{1}, b_{2}$ for $k=3$. The system

$$
\begin{aligned}
& 0=1+\sum_{j=1}^{3}\left(\left(\alpha_{j}-1\right)^{2}-\alpha_{j}^{2}\right)\left(\left(\beta_{j}-1\right)^{2}-\beta_{j}^{2}\right) \\
& 0=-2+\sum_{j=1}^{3}\left(\left(\alpha_{j}-1\right)^{3}-\alpha_{j}^{3}\right)\left(\left(\beta_{j}-1\right)^{3}-\beta_{j}^{3}\right) \\
& 0=3+\sum_{j=1}^{3}\left(\left(\alpha_{j}-1\right)^{4}-\alpha_{j}^{4}\right)\left(\left(\beta_{j}-1\right)^{4}-\beta_{j}^{4}\right) \\
& 0=-4+\sum_{j=1}^{3}\left(\left(\alpha_{j}-1\right)^{5}-\alpha_{j}^{5}\right)\left(\left(\beta_{j}-1\right)^{5}-\beta_{j}^{5}\right) \\
& 0=5+\sum_{j=1}^{3}\left(\left(\alpha_{j}-1\right)^{6}-\alpha_{j}^{6}\right)\left(\left(\beta_{j}-1\right)^{6}-\beta_{j}^{6}\right) \\
& 0=-6+\sum_{j=1}^{3}\left(\left(\alpha_{j}-1\right)^{7}-\alpha_{j}^{7}\right)\left(\left(\beta_{j}-1\right)^{7}-\beta_{j}^{7}\right)
\end{aligned}
$$

has (the unique) solution

$$
\begin{aligned}
\alpha_{1}= & -0.25815871587916770707043092744853466448964330026005 \ldots \\
& +i \cdot 0.48397242106377239253881674751055982149144184289297 \ldots \\
\alpha_{2}= & 0.038201465324611971071624393136243082171731381749928 \ldots \\
\alpha_{3}= & -0.25815871587916770707043092744853466448964330026005 \ldots \\
& -i \cdot 0.48397242106377239253881674751055982149144184289297 \ldots
\end{aligned}
$$$$
\beta_{1}=-0.38406133637441025553569425604004004944633805438883 \ldots
$$$$
\beta_{2}=-0.29702731502965659376677160286039309868060858219067 \ldots
$$$$
-i \cdot 0.49547669307316290426997439537218740680230881850428 \ldots
$$

$$
\begin{aligned}
\beta_{3}= & -0.29702731502965659376677160286039309868060858219067 \ldots \\
& +i \cdot 0.49547669307316290426997439537218740680230881850428 \ldots
\end{aligned}
$$


It follows that

$$
\begin{aligned}
-a_{2} & =\alpha_{1}+\alpha_{2}+\alpha_{3} \\
& =-0.47811596643372344306923746176082624680755521877097 \ldots \\
a_{1} & =\alpha_{1} \alpha_{2}+\alpha_{2} \alpha_{3}+\alpha_{3} \alpha_{1} \\
& =0.28115114446890147824727263979600428198559039680544 \ldots \\
-a_{0} & =\alpha_{1} \alpha_{2} \alpha_{3} \\
& =0.011493874548781090837165603520743707659595510062767 \ldots \\
-b_{2} & =\beta_{1}+\beta_{2}+\beta_{3} \\
& =-0.97811596643372344306923746176082624680755521877081 \ldots \\
b_{1} & =\beta_{1} \beta_{2}+\beta_{2} \beta_{3}+\beta_{3} \beta_{1} \\
& =0.56187579435242986644855803734308407205603467285750 \ldots \\
-b_{0} & =\beta_{1} \beta_{2} \beta_{3} \\
& =-0.12816986295374145841435561061729286028351448912202 \ldots
\end{aligned}
$$

Next, the continued fraction algorithm applied to the above numerical values, gives

$$
\begin{aligned}
& a_{2}=-<-1,1,1,10,1,12,7,1,1,5,1,4>=\frac{68143}{142524} \\
& a_{1}=<0,3,1,1,3,1,9,10,3,1,3,1,10>=\frac{186997}{665112} \\
& a_{0}=-<0,87,349,4,1,5,3,2>=-\frac{74381}{6471360} \\
& b_{2}=-<-1,45,1,2,3,1,1,7,2,2,3>=\frac{139405}{142524} \\
& b_{1}=<0,1,1,3,1,1,5,1,2,3,1,1,8,1,21,1,3>=\frac{1121131}{1995336} \\
& b_{0}=-<-1,1,6,1,4,18,2,3,1,1,4,1,8,1,5,3,1,1,2,3>=\frac{30689033}{239440320} .
\end{aligned}
$$

Then we have

$$
\lim _{n \rightarrow \infty} n^{7} M_{n}=\frac{\theta}{7}
$$


with

$$
\begin{aligned}
\theta & =\frac{1}{8}\left(7+\sum_{j=1}^{3}\left(\left(\alpha_{j}-1\right)^{8}-\alpha_{j}^{8}\right)\left(\left(\beta_{j}-1\right)^{8}-\beta_{j}^{8}\right)\right) \\
& =-0.01832772866046807884381439244713734156447898226906 \ldots \\
& =<-1,1,53,1,1,3,1,1,10,7,1,1,1,3,2,3,4,1,2,1,36,1,8,1,3,3> \\
& =-\frac{10833071983}{591075532800} .
\end{aligned}
$$

It is to be noticed that these results were rediscovered by us using Lemma 1 presented in the first part of this paper. We omit the proof for sake of simplicity.

Finally, remark that if the polynomials $P$ and $Q$ of $k$-th degree are already determined, say using the previous numerical method, then the problem of verifiying the speed of convergence of the corresponding sequence $M_{n}$ using Lemma 1 becomes a much easier task.

Acknowledgements. The author thanks the editors and the anonymous referee for useful ideas which improved much the initial form of this paper.

\section{REFERENCES}

[1] H. Alzer, Inequalities for the gamma and polygamma functions. Abh. Math. Sem. Univ. Hamburg, 68 (1998), 363-372.

[2] G.D. Anderson, R.W. Barnard, K.C. Richards, M.K. Vamanamurthy and M. Vuorinen, Inequalities for zero-balanced hypergeometric functions. Trans. Amer. Math. Soc., 347(5) (1995), 1713-1723.

[3] D.W. DeTemple, A quicker convergence to Euler's constant. Amer. Math. Monthly, 100(5) (1993), 468-470.

[4] D.W. DeTemple, A geometric look at sequences that converge to Euler's constant. College Math. J., 3 (2006), 128-131.

[5] C. Elsner, On a sequence transformation with integral coefficients for Euler's constant. Proc. Amer. Math. Soc., 123(5) (1995), 1537-1541.

[6] C. Elsner, On arithmetic properties of the convergents of Euler's number. Colloq. Math., 79(1) (1999), 133-145.

[7] C. Elsner, On a sequence transformation with integral coefficients for Euler's constant, II. J. Number Th., 124(2) (2007), 442-453.

[8] Kh. Hessami Pilehrood and T. Hessami Pilehrood, Approximations to Euler's constant, available online: http://arxiv.org/abs/0708.2771. 
[9] Kh. Hessami Pilehrood and T. Hessami Pilehrood, Arithmetical properties of some series with logarithmic coefficients. Math. Zeitschrift, 255(1) (2007), 117-131.

[10] Kh. Hessami Pilehrood, T. Hessami Pilehrood and W. Zudilin, Irrationality of certain numbers that contain values of the di- and trilogarithmhttp. Math. Zeitschrift, 254(2) (2006), 299-313.

[11] T. Hessami Pilehrood and Kh. Hessami Pilehrood, Criteria for irrationality of generalized Euler's constant. J. of Number Theory, 108(1) (2004), 169-185.

[12] M. Hindry and T. Rivoal, Le Lambda-calcul de Golomb et la conjecture de Bateman-Horn. Enseign. Math., 51 (2005), 265-318.

[13] C. Mortici, New approximations of the gamma function in terms of the digamma function. Appl. Math. Lett., 23(1) (2010), 97-100.

[14] C. Mortici and A. Vernescu, An improvement of the convergence speed of the sequence $\left(\gamma_{n}\right)_{n \geq 1}$ converging to Euler's constant. An. Şt. Univ. Ovidius Constanţa, 13(1) (2005), $97-100$.

[15] C. Mortici and A. Vernescu, Some new facts in discrete asymptotic analysis. Math. Balkanica, 21(3-4) (2007), 301-308.

[16] C. Mortici, Product approximations via asymptotic integration. Amer. Math. Monthly, 117(5) (2010), 434-441.

[17] C. Mortici, Optimizing the rate of convergence in some new classes of sequences convergent to Euler's constant. Anal. Appl. (Singap.), 8(1) (2010), 99-107.

[18] C. Mortici, Improved convergence towards generalized Euler-Mascheroni constant. Appl. Math. Comput., 215(9) (2010), 3443-3448.

[19] C. Mortici, A quicker convergence toward the gamma constant with the logarithm term involving the constant e. Carpathian J. Math., 26(1) (2010), 86-91.

[20] T. Rivoal, Rational approximations for values of derivatives of the Gamma function. Trans. Amer. Math. Soc., 361 (2009), 6115-6149.

[21] T. Rivoal, Convergents and irrationality measures of logarithms. Rev. Mat. Iberoamericana, 23(3) (2007), 931-952.

[22] J. Sondow, Criteria for irrationality of Euler's constant. Proc. Amer. Math. Soc., 131 (2003), 3335-3344.

[23] J. Sondow, Double integrals for Euler's constant and $\ln 4 / \pi$ and an analog of Hadjicostas's formula. Amer. Math. Monthly, 112 (2005), 61-65.

[24] J. Sondow and W. Zudilin, Euler's constant, $q$-logarithms, and formulas of Ramanujan and Gosper. Ramanujan J., 12 (2006), 225-244.

[25] J. Sondow, An infinite product for $e^{\gamma}$ via hypergeometric formulas for Euler's constant, $\gamma$, available online at: http://arxiv.org/abs/math.CA/0306008.

[26] J. Sondow, New Vacca-type rational series for Euler's constant and its “alternating” analog $\ln 4 / \pi$, available online at: http://arxiv.org/abs/math.NT/0508042.

[27] S.R. Tims and J.A. Tyrrell, Approximate evaluation of Euler's constant. Math. Gaz., 55, 391 (1971), 65-67.

[28] R.M. Young, Euler's Constant. Math. Gaz., 75 (1991), 187-190. 